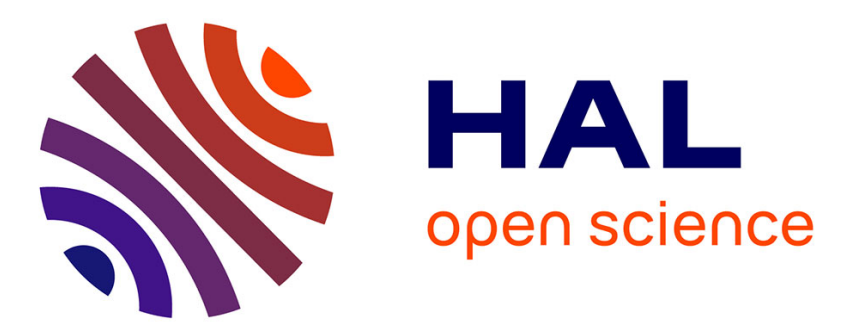

\title{
Structure of the (010) surface of the orthorhombic complex metallic alloy $\mathrm{T}-\mathrm{Al} 3(\mathrm{Mn}, \mathrm{Pd})$
}

Th. Deniozou, R. Addou, A.K. Shukla, M. Heggen, M. Feuerbacher, M. Krajčí, J. Hafner, R. Widmer, O. Gröning, Vincent Fournée, et al.

\section{- To cite this version:}

Th. Deniozou, R. Addou, A.K. Shukla, M. Heggen, M. Feuerbacher, et al.. Structure of the (010) surface of the orthorhombic complex metallic alloy T -Al 3 (Mn,Pd). Physical Review B: Condensed Matter and Materials Physics (1998-2015), 2010, 81 (12), 10.1103/PhysRevB.81.125418 . hal-01665178

\section{HAL Id: hal-01665178 https://hal.science/hal-01665178}

Submitted on 15 Dec 2017

HAL is a multi-disciplinary open access archive for the deposit and dissemination of scientific research documents, whether they are published or not. The documents may come from teaching and research institutions in France or abroad, or from public or private research centers.
L'archive ouverte pluridisciplinaire HAL, est destinée au dépôt et à la diffusion de documents scientifiques de niveau recherche, publiés ou non, émanant des établissements d'enseignement et de recherche français ou étrangers, des laboratoires publics ou privés. 


\title{
Structure of the (010) surface of the orthorhombic complex metallic alloy $\mathrm{T}-\mathrm{Al}_{3}(\mathrm{Mn}, \mathrm{Pd})$
}

\author{
Th. Deniozou, * R. Addou, and A. K. Shukla \\ Institut Jean Lamour, UMR 7198, CNRS-Nancy-Université-UPV-Metz, Ecole des Mines, Parc de Saurupt, 54042 Nancy Cedex, France
}

\author{
M. Heggen and M. Feuerbacher \\ Institut für Festkörperforschung, Forschungszentrum Jülich, 52425 Jülich, Germany
}

M. Krajčí and J. Hafner

Institute of Physics, Slovak Academy of Sciences, Dúbravská cesta 9, SK-84511 Bratislava, Slovak Republic

and Fakultät für Physik and Center for Computational Materials Science, Universität Wien, Sensengasse 8/12, A-1090 Wien, Austria

R. Widmer and O. Gröning

EMPA, Swiss Federal Laboratories for Materials Testing and Research, nanotech@surfaces Laboratory, Feuerwerkerstrasse 39, CH-3602 Thun, Switzerland

\author{
V. Fournée, J.-M. Dubois, and J. Ledieu ${ }^{\dagger}$ \\ Institut Jean Lamour, UMR 7198, CNRS-Nancy-Université-UPV-Metz, Ecole des Mines, Parc de Saurupt, 54042 Nancy Cedex, France
}

(Received 9 September 2009; revised manuscript received 9 February 2010; published 17 March 2010)

\begin{abstract}
The atomic and electronic structures of the (010) surface of the $\mathrm{T}_{-} \mathrm{Al}_{3}(\mathrm{Mn}, \mathrm{Pd})$ complex metallic alloy is investigated by means of low-energy electron diffraction (LEED), scanning tunneling microscopy (STM), $\mathrm{x}$-ray and ultraviolet photoelectron spectroscopy (XPS and UPS), x-ray photoelectron diffraction (XPD), and ab initio calculations. While structural imperfections are observed at the surface and out of the various possible terminations, the puckered P2 layer is identified as the only surface termination, thus pointing out the existence of a well-defined minimum in the surface energy landscape. The measured step heights correspond to distances between identical planes along the [010] direction in the bulk model, i.e., $b / 2$. A bias dependency of the STM topography is found. The XPD and LEED patterns confirm the pseudotenfold symmetry of the sample. XPS and UPS show a more metallic signature of the T phase compared to Al-based quasicrystalline phases.
\end{abstract}

DOI: 10.1103/PhysRevB.81.125418

PACS number(s): 61.44.Br, 68.35.bd, 68.37.Ef

\section{INTRODUCTION}

Complex metallic alloys (CMA) are a class of intermetallic compounds which encompass quasicrystals and their approximant phases. ${ }^{1}$ The structure of CMA is best described by highly symmetric clusters which decorate a large unit cell containing up to some thousands of atoms. Quasicrystals represent the ultimate case where the unit cell is considered as infinite. Within the large family of CMA, the Al-Pd-Mn system has attracted much interest. This is partly motivated by the presence in the phase diagram of stable and metastable quasicrystalline phases and orthorhombic phases which possess "giant" unit cells. The latter are periodic phases and were identified as approximants of either icosahedral or decagonal quasicrystals. ${ }^{2}$ Among the various approximants, the $\xi$ and $\xi^{\prime}$ phases are situated on the Pd-rich side of the ternary phase diagram while the Taylor's $(\mathrm{T})$ or Robinson's $(R)$ orthorhombic phases, which are related to the decagonal state, are found in the Mn-rich region. ${ }^{3-5}$ For instance, the orthorhombic $\mathrm{T}-\mathrm{Al}_{3} \mathrm{Mn}$ crystal ${ }^{3}$ with a $b$ unit-cell parameter equal to $12.43 \AA$ parallel to the pseudotenfold axis $(p$-10f) is closely related to the decagonal Al-Pd-Mn quasicrystal of similar periodicity along the tenfold axis. ${ }^{6,7}$ The structure within the planes perpendicular to the $p$-10f axis of both $\mathrm{T}$ and $R$ phases can be understood as a periodic stacking of two different tilings based on a single tile $\mathrm{H}$ with the shape of a squashed hexagon. While the $R$ phase can be seen as a parallel packing of the tiles, in the $\mathrm{T}$ phase the tiles form a "zigzag" pattern. In the structurally more complex decagonal phase, additional tiles are required. ${ }^{6,8}$

Approximant phases have often been introduced to model the physical and chemical properties of their parent quasicrystal and to perform electronic-structure calculations requiring a periodic cell. ${ }^{8-10}$ While the surface of the $i$-Al-Pd-Mn quasicrystal has been intensively investigated, ${ }^{11}$ only a limited number of experimental surface studies have been reported on approximant phases in the Al-Pd-Mn system. The $\mathrm{T}-\mathrm{Al}_{3} \mathrm{Mn}$ phase has been fortuitously observed using scanning tunneling microscopy (STM) on the fivefold surface of the Al-Pd-Mn quasicrystal as a consequence of the decomposition of the sample at elevated temperature. ${ }^{12}$ To our knowledge, the work reported by Fournée et al. ${ }^{13}$ on the $\xi^{\prime}$-Al-Pd-Mn phase marks the first surface study carried out on single crystals of approximants. Two sets of $\xi^{\prime}$ samples were produced either by Bridgman or self-flux technique. Following a similar preparation, both surfaces exhibit a $p$-10f low-energy electron diffraction (LEED) pattern. Using STM, differences in the local atomic arrangements have been identified on terraces between both samples. However, the STM images can be interpreted for both cases as a representation of planes perpendicular to the $p$-10f axis of the bulk model derived by Boudard et al. ${ }^{14}$ In the same study and in parallel, several crystalline phases were induced on the fivefold surface of the $i$-Al-Pd-Mn quasicrystal by sputter annealing at "low" and "high" temperatures compared to the optimized temperature range known to produce bulklike ter- 
minated quasicrystal surfaces. ${ }^{15,16}$ While the lowtemperature phase could not be identified with a known bulk phase, the unit-cell parameters of the high-temperature phase matched the lattice parameters of the $\mathrm{T}-\mathrm{Al}_{72.3} \mathrm{Pd}_{3.2} \mathrm{Mn}_{24.5}$ approximant [labeled $\mathrm{T}-\mathrm{Al}_{3}(\mathrm{Mn}, \mathrm{Pd})$ hereafter]. As a consequence of the high annealing treatment, the chemical composition in the near-surface region could have been shifted out of the stability range of the icosahedral phase, leading to the formation of this T phase. ${ }^{13}$ The limited resolution of the STM images precluded the identification of the surface structure. It was speculated that it should correspond to a particular pair of layers related by a mirror plane. The atomic structure of this approximant is isomorphic to the $\mathrm{T}-\mathrm{Al}_{3} \mathrm{Mn}$ phase and has been described by Klein et al. ${ }^{17}$

In this paper, we report a study of the (010) surface of the orthorhombic $\mathrm{T}_{-} \mathrm{Al}_{3}(\mathrm{Mn}, \mathrm{Pd})$ single crystal using $\mathrm{STM}$, LEED, $x$-ray photoelectron diffraction (XPD), and ultraviolet and x-ray photoelectron spectroscopy (UPS and XPS). The model of the bulk structure of the $\mathrm{T}-\mathrm{Al}_{3}(\mathrm{Mn}, \mathrm{Pd})$ approximant is presented in Sec. II. The experimental details and the surface preparation are described in Sec. III. Following the description of the computational details in Sec. IV, we present atomically resolved STM images and identify the surface structure in Sec. V.

$A b$ initio density-functional methods (see Sec. IV) have been used to calculate the simulated STM images, the electronic structure, and the simulated photoemission spectra.

The bias dependency observed in STM images is also confirmed by simulated STM images. The electronic structure of several complex metallic alloys and, in particular, of the Al-Pd-Mn quasicrystal is characterized by a reduced density of states at the Fermi level $\left(E_{F}\right)$, termed a pseudogap. ${ }^{18}$ It has been found to play a critical part in the alloy stability and in transport properties. ${ }^{9,19,20}$ On valence-band spectra, it manifests itself by a decrease in the spectral intensity in the vicinity of $E_{F}$ compared to the sharp Fermi edge observed for metals. The asymmetry of core-level line shapes can also be indicative of the density of states near $E_{F} \cdot{ }^{21,22}$ This is the case for the Mn $2 p$ core level in the $i$-Al-Pd-Mn, which displays a particularly low-asymmetry effect related to the presence of a pseudogap at $E_{F} \cdot{ }^{23,24}$ Hence, a comparison of the Mn $2 p$ core level and of the valence-band region close to the Fermi edge between the $i$-Al-Pd-Mn quasicrystal and the $\mathrm{T}-\mathrm{Al}_{3}(\mathrm{Mn}, \mathrm{Pd})$ crystal will be presented in Sec. V. Finally, the results will be discussed in Sec. VI.

\section{MODEL OF THE BULK STRUCTURE}

As for the $\mathrm{T}_{-} \mathrm{Al}_{3} \mathrm{Mn}$ phase, ${ }^{3}$ the orthorhombic $\mathrm{T}-\mathrm{Al}_{3}(\mathrm{Mn}, \mathrm{Pd})$ crystal is an approximant of the stable decagonal Al-Pd-Mn quasicrystal. Its atomic structure was determined by single-crystal $\mathrm{x}$-ray diffraction with an atomic composition of $\mathrm{Al}_{72.3} \mathrm{Pd}_{3.2} \mathrm{Mn}_{24.5}{ }^{17,25}$ The space group is Pnma, the unit cell contains 156 atoms and the lattice parameters are $a=14.717 \AA, b=12.510 \AA$, and $c=12.594 \AA$.

According to Klein et al. ${ }^{17}$ the bulk structure of this approximant (see Fig. 1) is described along the $\mathbf{b}$ axis by two types of layers, a flat $(\mathrm{F})$ layer and a puckered $(\mathrm{P})$ layer which can be divided into two sublayers $\mathrm{P} 1$ and $\mathrm{P} 2$. The F

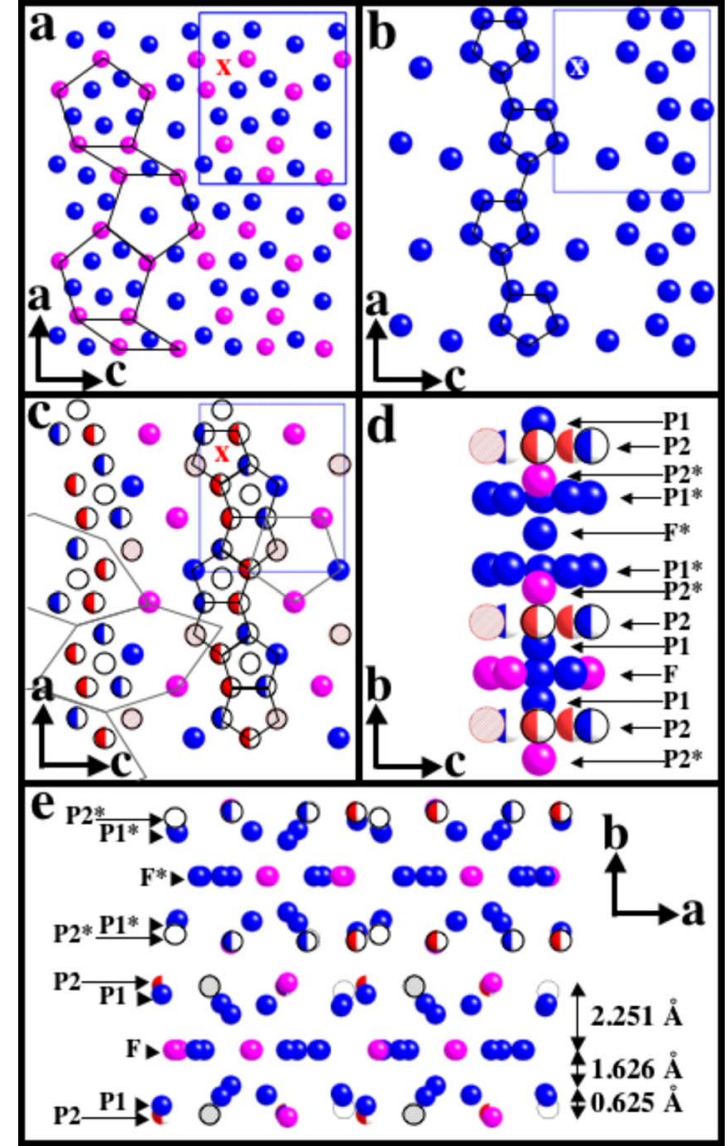

FIG. 1. (Color online) $[(\mathrm{a})-(\mathrm{c})]$ The structure of the orthorhombic $\mathrm{T}_{-} \mathrm{Al}_{3}(\mathrm{Mn}, \mathrm{Pd})$ (Refs. 17 and 25) is presented along the [010] direction. Blue (dark gray) spheres correspond to $\mathrm{Al}$ atoms, pink (light gray) spheres to $\mathrm{Mn}$ atoms, and others to mixed atomic sites. The orthorhombic unit cell is outlined by a rectangle. (a) Representation of the flat layer (f). Connecting the Mn atoms leads to a tiling composed of pentagons and rhombi. (b) The puckered layer (P1) is composed only of $\mathrm{Al}$ atoms arranged in almost perfect pentagonal chains. (c) The second puckered layer (P2) is composed of Al, Pd, and $\mathrm{Mn}$ atoms and several positions have a mixed occupancy. The blue (black) and white spheres correspond to mixed $\mathrm{Mn}-\mathrm{Al}$ $(0.5 / 0.5)$ sites [referred as $\mathrm{TM}(1)$ and $\mathrm{TM}(2)$ sites by Klein et al. (Ref. 17)], the red (gray) and white spheres correspond to mixed Mn-Al (0.3/0.7) sites [TM(3) and TM(4)], the shaded spheres correspond to mixed $\mathrm{Mn}-\mathrm{Al}(0.15 / 0.85)$ sites $[\mathrm{TM}(5)]$, and the empty circles correspond to mixed Pd-Al (0.625/0.375) sites [TM(6)]. Mn atoms are organized as a zigzag line while other sites form pentagonal chains. Several H tiles are also drawn. (d) Schematic view along the [010] direction of one column of interpenetrating icosahedral clusters centered at point $x$ marked in (a)-(c). (e) Representation of the stacking of layers perpendicular to the $\mathbf{b}$ axis in the unit cell of the model structure.

layer located at $y=1 / 4$ contains a maximum of 22 atoms per unit cell with $14 \mathrm{Al}$ and $8 \mathrm{Mn}$ atoms. As shown in Fig. 1(a), the interconnection of $\mathrm{Mn}$ atoms leads to the formation of a tiling composed of pentagons and rhombi. The puckered layer $\mathrm{P} 1$ located at $y \approx 0.38$ contains $12 \mathrm{Al}$ atoms arranged in pentagonal chains [Fig. 1(b)]. The atomic positions of both $\mathrm{F}$ and P1 layers are all fully occupied while this is not the case for the P2 plane. The puckered layer P2 located at $y \approx 0.43$ 
contains 16 atoms and all three elements. Four positions are fully occupied by two $\mathrm{Al}$ and two Mn atoms [blue (black)/ pink (gray) spheres on Fig. 1(c)]. In addition, there are ten mixed Al-Mn sites and two mixed Pd-Al sites. When linked, the $\mathrm{Al}$ and mixed sites form pentagonal chains while fully occupied Mn sites form zigzag chains [Fig. 1(c)]. ${ }^{17}$ Along the [010] direction, the atomic structure can be viewed as columns of interpenetrating icosahedral clusters [see Fig. $1(d)$ ] resulting from the alternate stacking of pentagonal prisms $(p)$ and antiprisms $(a)$, each separated by one atom in the sequence apaapa. ${ }^{6}$ The columns intersect F, P1, and P2 planes at a position labeled $x$ in Figs. 1(a)-1(c). As shown in Fig. 1(e), the stacking layers along the [010] direction has the following sequence:

\section{P2P1FP1P2P2*P1 ${ }^{*}{ }^{*} \mathrm{P} 1{ }^{*} \mathrm{P} 2 *$}

where $\mathrm{F}(y=0.25)$ and $\mathrm{F}^{*}(y=0.75)$ are mirror planes. $\mathrm{P} 1^{*}$, $\mathrm{P} 2^{*}$, and $\mathrm{F}^{*}$ have a twofold rotational symmetry against $\mathrm{P} 1$, $\mathrm{P} 2$, and $\mathrm{F}$. The distance between the different layers are $d_{\mathrm{P} 1 \mathrm{P} 2}=0.625 \AA, d_{\mathrm{FP} 1}=1.626 \AA$, and $d_{\mathrm{FP} 2}=2.251 \AA .{ }^{17}$

The unit cell thus consists of two blocks of planes (P2P1FP1P2) and $\left(\mathrm{P} 2{ }^{*} \mathrm{P} 1{ }^{*} \mathrm{~F}^{*} \mathrm{P} 1{ }^{*} \mathrm{P} 2 *\right)$ related by a twofold symmetry. The thickness of each block is $b / 2$.

\section{EXPERIMENTAL DETAILS}

The $\mathrm{T}-\mathrm{Al}_{3}(\mathrm{Mn}, \mathrm{Pd})$ crystal with the chemical composition $\mathrm{Al}_{78} \mathrm{Pd}_{4} \mathrm{Mn}_{18}$ (at.\%) used in this experiment has been grown by the self-flux technique. The high-purity starting materials were sealed in an alumina crucible under protective argon atmosphere. Crystal growth was performed in a chamber furnace at a cooling rate of $1 \mathrm{~K} / \mathrm{h}$. After growth, the crystals were annealed for $120 \mathrm{~h}$ at $1073 \mathrm{~K}$. The high structural quality of the material was characterized by phasecontrast optical microscopy, scanning electron microscopy, and transmission electron microscopy. A sample for surface investigation was oriented using backreflection Laue x-ray diffraction and was cut perpendicular to its [010] direction. The surface has been mechanically polished using diamond paste with decreasing grain size down to $0.25 \mu \mathrm{m}$ and using Syton ${ }^{\circledR}$ for the final polishing cycles. At this stage, the sample appearance is mirrorlike. The sample is investigated under UHV condition with a base pressure below $5 \times 10^{-11}$ mbar. The preparation of the crystal consists of cycles of $\mathrm{Ar}^{+}$-ion sputtering at $2 \mathrm{keV}$ for $30 \mathrm{~min}$ and annealing to temperatures ranging from 853 to $923 \mathrm{~K}$ from $40 \mathrm{~min}$ to $2 \mathrm{~h}$. The temperature of the sample is monitored using an infrared optical pyrometer with the emissivity set to 0.35 coupled with a K-type thermocouple. This preparation method results in a step-terrace morphology of the surface. In the chosen temperature range, no significant changes in composition, surface structure, and morphology are found. The composition and electronic structure of the sample are monitored using x-ray (resolution $0.8 \mathrm{eV}$ ) and ultraviolet (resolution $100 \mathrm{meV}$ ) photoelectron spectroscopy while the overall surface structure is assessed by LEED. The STM measurements have been performed at room temperature using an Omicron variable temperature AFM/STM (VT-AFM). The XPD measurements have been carried out in a separate
UHV chamber (EMPA Thun) using a nonmonochromatised Al $K \alpha$ radiation and a modified Omicron photoelectron spectrometer equipped with an EA 125 HR electron analyzer operated in constant analyzer energy mode. The calibration of the spectrometer has been done with respect to the $\mathrm{Au} 4 f_{7 / 2}$ binding energy of $83.8 \mathrm{eV}$. The structural quality of the surface is monitored using LEED technique prior to XPD measurements.

\section{COMPUTATIONAL DETAILS}

The theoretical $a b$ initio investigations are based on density-functional theory (DFT). In our previous study ${ }^{8}$ of the approximant phases in decagonal Al-Pd-Mn we used the linear muffin-tin orbital method to calculate the electronic structure. In the present work we have used the Vienna $a b$ initio simulation package (VASP) (Refs. 26 and 27) to perform electronic-structure calculations and structural optimizations. VASP performs an iterative solution of the KohnSham equations within a plane-wave basis. A semilocal gradient-corrected functional ${ }^{28}$ (PW91) is used to describe electronic exchange and correlation. The projectoraugmented wave method ${ }^{27,29}$ is used to describe the electronion interactions. The essential input information for the calculation of simulated STM images is the energy-resolved charge-density distributions. STM images of surfaces have been calculated according to the Tersoff-Hamann approximation. ${ }^{30}$

As the bulk structure model of Klein et al. ${ }^{17}$ contains fractional and mixed occupancies of the sites, it cannot be directly used for the $a b$ initio calculations. Instead we have used a model derived by Mihalkovič and published in the alloy database (Ref. 31). The model exhibits optimized geometry and chemical decorations of the sites. The sites with fractional and mixed $\mathrm{Pd}-\mathrm{Al}$ and $\mathrm{Mn}-\mathrm{Al}$ occupancies were resolved with respect to the Pnma space-group symmetry of the unit cell. The cell parameters of $a=14.784 \AA$, $b=12.496 \AA$, and $c=12.606 \AA$ are in good agreement with the experimental data. The composition of the model is $\mathrm{Al}_{79.5} \mathrm{Mn}_{15.4} \mathrm{Pd}_{5.1}$, i.e., closer to the bulk composition of the sample used in this study. A calculation of the heat of formation has confirmed that the optimized model represents a stable phase in the Al-Mn-Pd phase diagram.

A model for the surface is constructed by cutting a thick slab from the bulk structure. The thickness of the slab must be large enough to stabilize the surface. Our model of the surface consists of a slab with the thickness of a single unit cell and a vacuum layer of $10 \AA$. The position of atoms in the model were relaxed by the Hellmann-Feynman interatomic forces. The positions of atoms in the top five atomic planes were free to relax, the atoms in the bottom five planes were kept at their bulk positions.

\section{RESULTS}

\section{A. Identification of the surface structure}

After annealing the $\mathrm{T}-\mathrm{Al}_{3}(\mathrm{Mn}, \mathrm{Pd})$ sample to $863 \mathrm{~K}$ for 2 $\mathrm{h}$, the surface is well ordered as indicated by the sharp LEED patterns shown in Fig. 2. The latter are dominated by almost 


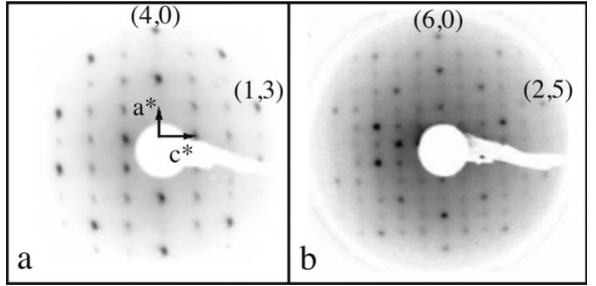

FIG. 2. [(a) and (b)] LEED patterns (inverted for clarity) recorded on the clean surface at $25 \mathrm{eV}$ and $55.9 \mathrm{eV}$, respectively. The pseudotenfold symmetry is made evident on both patterns by the presence of decagonal rings consisting of ten darker diffraction spots.

perfect decagonal rings made of ten bright diffraction spots of equal intensity. For the LEED pattern recorded at $55.9 \mathrm{eV}$ [see Fig. 2(b)], one can distinguish an inner and an outer decagonal ring. Because all spots are periodically spaced, the symmetry of the LEED pattern is referred as $p$-10f. The surface structure is orthorhombic with the axial ratio $\left(a=14.58 \pm 0.43 \AA\right.$ and $c=12.40 \pm 0.42 \AA$ giving $\left.\frac{a}{c} \approx 1.18\right)$ in agreement, within the accuracy of our measurements, to that reported by Klein et al. ${ }^{17}\left(\frac{a}{c}=1.17\right)$. The reciprocal lattice vectors of the orthorhombic lattice are outlined on the LEED pattern shown in Fig. 2(a).

At this stage, the STM investigation of the $\mathrm{T}-\mathrm{Al}_{3}(\mathrm{Mn}, \mathrm{Pd})$ sample surface reveals a step-terrace morphology. The width of the atomically flat terraces ranges from 0.1 to $0.3 \mu \mathrm{m}$ [Figs. 3(a) and 3(b)]. Based on a qualitative comparison to other approximant samples $\left(\mathrm{Al}_{13} \mathrm{Co}_{4},{ }^{32} \xi^{\prime}-\right.$ and T-phase

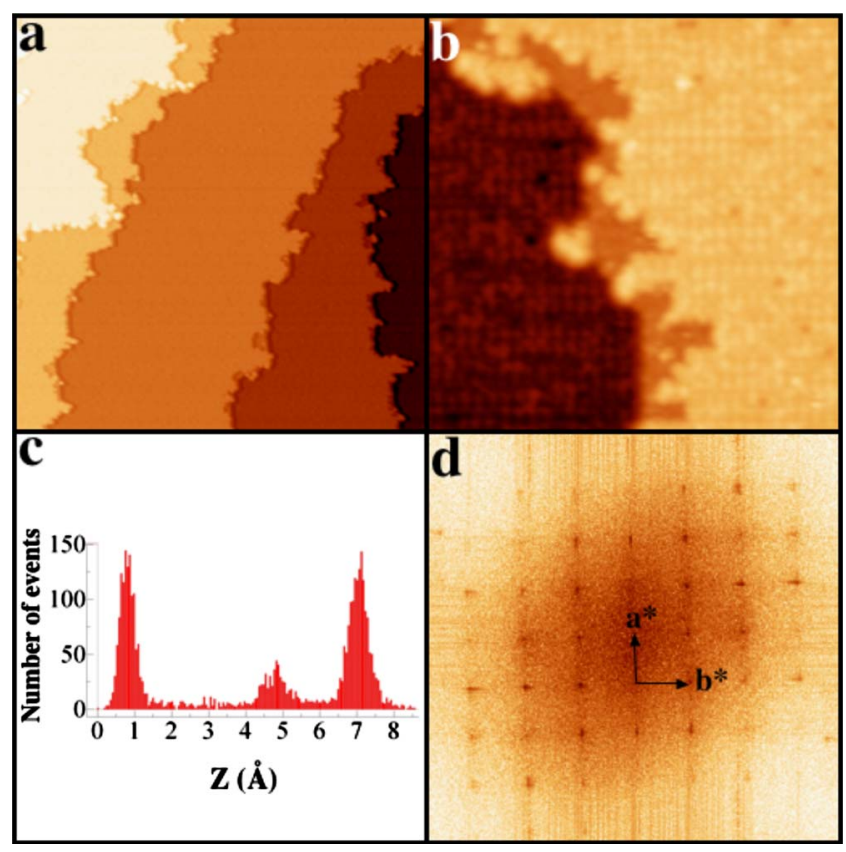

FIG. 3. (Color online) (a) STM image $\left(250 \times 250 \mathrm{~nm}^{2}\right)$ showing the step and terrace morphology. (b) STM image $\left(40 \times 40 \mathrm{~nm}^{2}\right)$ showing a narrow terrace on the step edge. (c) The height histogram calculated from a $20 \times 20 \mathrm{~nm}^{2}$ STM image exhibits the three possible step heights observed across terraces. (d) Fast Fourier transform calculated from a $50 \times 50 \mathrm{~nm}^{2}$ high-resolution STM image.

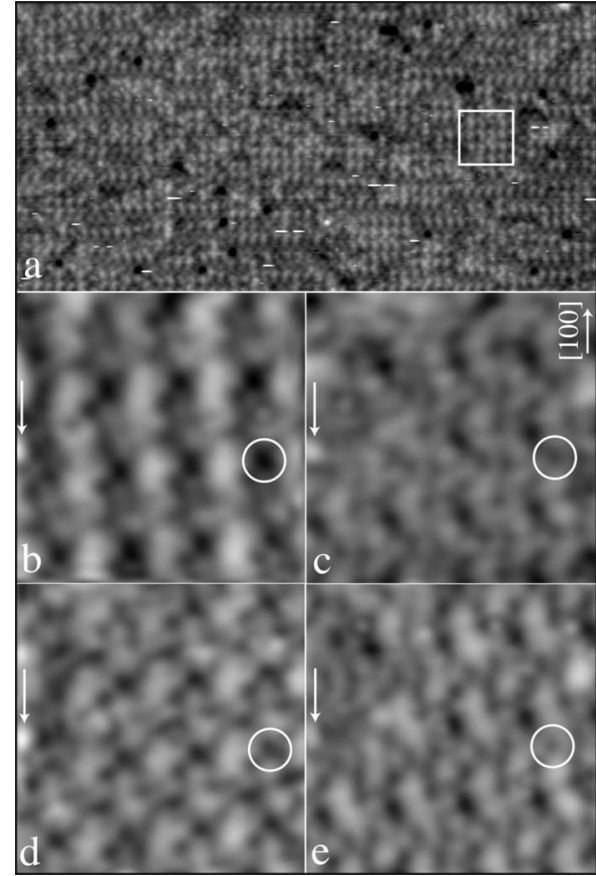

FIG. 4. (a) $70 \times 35 \mathrm{~nm}^{2}$ STM image recorded on a terrace for $V_{b}=2 \mathrm{~V}$ and $I_{t}=0.06 \mathrm{nA}$. The white frame outlines a patch of homogeneous contrast. [(b)-(e)] STM images $\left(5.1 \times 5.1 \mathrm{~nm}^{2}\right)$ acquired on the same region using a constant current of $40 \mathrm{pA}$ and a bias of (b) $0.8 \mathrm{~V}$, (c) $-0.8 \mathrm{~V}$, (d) $0.4 \mathrm{~V}$, and (e) $-0.4 \mathrm{~V}$. The arrows point to a common defect visible on all images. As shown by the circles located at the same position on all images, the contrast varies drastically with the applied bias.

approximants, ${ }^{13,33}$ for instance), the roughness of the step edges observed on the $\mathrm{T}-\mathrm{Al}_{3}(\mathrm{Mn}, \mathrm{Pd})$ surface appears to be more pronounced. Height profiles measurements across several terraces exhibit a main step height of $6.3 \pm 0.3 \AA$, which corresponds to half of the lattice parameter along the [010] direction $(\simeq \mathbf{b} / 2)$. As shown in Fig. 3(b), an extra step height equal to $2.25 \pm 0.10 \AA$ is often observed on very narrow terraces. This specific height can be related to the $d_{\mathrm{FP} 2}(2.251 \AA)$ or to the $d_{\mathrm{P} 1{ }^{*} \mathrm{P} 2}(2.378 \AA)$ interlayer distances present in the bulk model. The roughness calculated on large terraces using the root-mean-square height $\left(Z_{\mathrm{rms}}\right)$ ranges from 0.1 to $0.2 \AA$ depending on the scan size and bias applied. These values are almost two times smaller than the $Z_{\text {rms }}$ values measured on quasicrystal surfaces. ${ }^{34}$ The fast Fourier transform (FFT) calculated from the clean surface displays an orthorhombic structure [Fig. 3(d)] with the lattice-parameter dimensions expected from that of the bulk structural model.

While the LEED patterns and the calculated FFT suggest a well-ordered surface, high-resolution STM images recorded on single terraces exhibit a high degree of structural imperfections. This is demonstrated in Fig. 4(a) which displays an STM image recorded on a single terrace. Here, the surface consists of areas of homogeneous contrast and structure. These well-ordered patches are distributed nonuniformly across the terrace but are in registry with the orthorhombic periodic lattice beneath. This topography is representative of what has been observed across the surface 


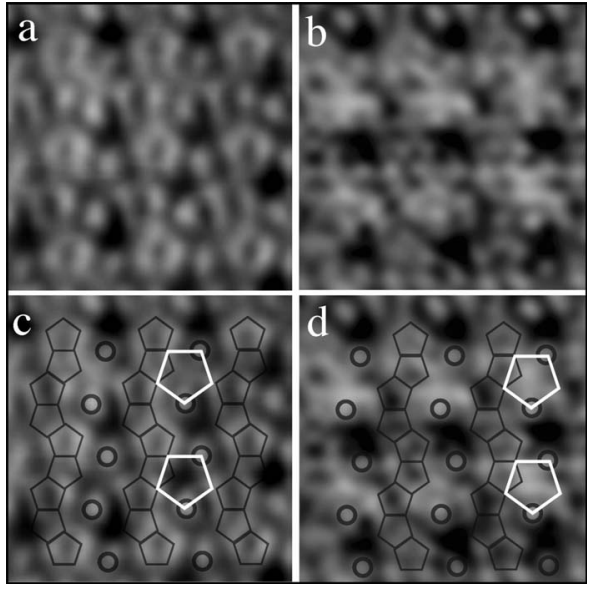

FIG. 5. [(a) and (c)] Atomically resolved STM image $\left(4 \times 4 \mathrm{~nm}^{2}\right)$ recorded from the clean surface at $V_{\text {bias }}=-0.4 \mathrm{~V}$ and $I_{t}=0.36 \mathrm{nA}$. This image has been filtered to emphasize the structure. [(b) and (d)] Atomically resolved STM image $\left(4 \times 4 \mathrm{~nm}^{2}\right)$ recorded on a different region of the same sample at $V_{\text {bias }}=0.6 \mathrm{~V}$ and $I_{t}=0.50 \mathrm{nA}$. This image has been rotated by $\pi$ for comparison purposes. [(c) and (d)] A tiling corresponding to the P2 layer has been superimposed on both images. The pentagonal chains arranged in a zigzag manner and individual $\mathrm{Mn}$ atoms are clearly discernable. While positioned identically with respect to the tiling, the contrast inside the largest pentagons varies drastically within the same image (c) as a consequence of compositional disorder, and between images (c) and (d) with the reversed sign of the bias voltage.

sample independently of the annealing time and temperatures used in our study. In addition to the difficulty introduced by the surface imperfections to interpret the surface structure, STM images are strongly bias dependent. The influence of the scanning parameters is demonstrated in Figs. 4(b)-4(e) where an identical surface region has been imaged for different bias values at a constant current of $40 \mathrm{pA}$. As it will be shown later, a much higher tunneling current is required to discern finer structures and to obtain atomic resolution. As a reference point, a common defect has been outlined by an arrow at the same position on all images. When scanning the unoccupied states near the Fermi level, the topography is dominated by broad and bright features which highlight the orthorhombic nature of the surface structure [see Fig. 4(b)]. In comparison, the STM images acquired at negative bias appear less corrugated and are dominated mainly by zigzag chains propagating in the [100] direction [Fig. 4(c)]. Darker contrasts usually associated with depressions are also distinguishable when probing both the occupied and unoccupied states. As demonstrated by the circles drawn at the same position in Figs. 4(b)-4(e), the depressions present in Fig. 4(b) almost disappear in Fig. 4(e) where new ones emerge at negative bias.

Using a higher tunneling current, the fine structural details of the experimental STM images are now comparable to those observed on simulated images (see Fig. 8). Figures 5(a) and 5(b) present two atomically resolved STM images recorded on two different regions on the $\mathrm{T}-\mathrm{Al}_{3}(\mathrm{Mn}, \mathrm{Pd})$ surface. Figure 5(b) has been acquired in a region referred as homogeneous in contrast to Fig. 5(a). Both images display pentagonal depressions resembling dark stars but Fig. 5(b) has been rotated by $\pi$ to display the pentagonal depressions in the same orientation as in Fig. 5(a). This effect is to be expected from the twofold rotational symmetry between $\mathrm{P} 1{ }^{*}$, $\mathrm{P}^{*}$, and $\mathrm{F}^{*}$ against $\mathrm{P} 1, \mathrm{P} 2$, and F, i.e., on adjacent terraces separated by a step height equal to $b / 2$. Figure 5(a) exhibits local pentagonal motifs and individual atoms arranged in a zigzag manner propagating along the [100] direction. These atomic features are less discernible in Fig. 5(b). Indeed, this latter STM image is dominated by brighter and more diffuse features [outlined by a white pentagon in Fig. 5(d)], which hinder the resolution of several pentagonal motifs and consequently of isolated atoms. To interpret these observations, the structures present on both STM images have been compared to individual planes present within the bulk model proposed by Klein et al. ${ }^{17}$ and described in Fig. 1. Because of the sizes and the arrangements of the pentagons in chains, the surface structures are compatible with both P1 and P2 puckered layers [Figs. 1(b) and 1(c)] but no agreement has been found with the $\mathrm{F}$ layer. To discriminate between the two possible puckered layers, one has to consider the alignment of isolated atoms (zigzag) with respect to the chain of pentagonal motifs. Hence, a tiling corresponding to the skeleton of the P2 structure has been superimposed on both images [Figs. 5(c) and 5(d)]. As shown in Fig. 5(c), the isolated atoms are aligned with shared edges of pointing "up" and "down" pentagons which belong to the chains propagating in the [100] direction. This local arrangement is consistent with the atomic positions present within the P2 layer but does not match those of the P1 layer. Based on these measurements, the surface structure shown in Figs. 5(a) and 5(b) is assigned to a P2-type termination.

We now analyze the origin of the contrast differences observed between both STM images. To this end, larger pentagons (outlined in white) have been placed on high-intensity patches observed in Fig. 5(d). The pentagon edge length is chosen such as to decorate two of its opposite vertices with the isolated atoms arranged in a zigzag manner, i.e., atomic positions fully occupied by $\mathrm{Mn}$ atoms in the P2 layer. A similar pentagon is drawn on the P2 structure presented in Fig. 1(c). The three remaining vertices correspond to vertices of smaller pentagons which form the chains propagating along the [100] direction. Two out of these three atomic positions correspond to mixed $\mathrm{Mn} / \mathrm{Al}$ sites referred as $\mathrm{TM}(3)$ and $\mathrm{TM}(1)$ by Klein et $a .^{17}$ with occupation factors of $0.3 \mathrm{Mn}-0.7 \mathrm{Al}$ and $0.5 \mathrm{Mn}-0.5 \mathrm{Al}$, respectively. The third one, which belongs to the adjacent pentagonal chain, is fully populated by an Al atom. The remaining vertex of the pentagon is populated by an Al atom. Similar "white" pentagons are positioned identically in Fig. 5(c) but, this time, dimmer and darker contrasts associated with depressions appear within the motifs. Consequently, Fig. 5(c) exhibits additional hollow sites which are not periodically distributed in the image. This approach allows us to pinpoint and to attribute the drastic variation in the intensity distribution between both STM images [Figs. 5(a) and 5(b)] to the decoration within these large pentagons. Along the pentagonal chains [see Fig. $5(d)]$, one of the vertices of the small pentagons (pointing down) lies alternatively inside the large white pentagons or 
(a)

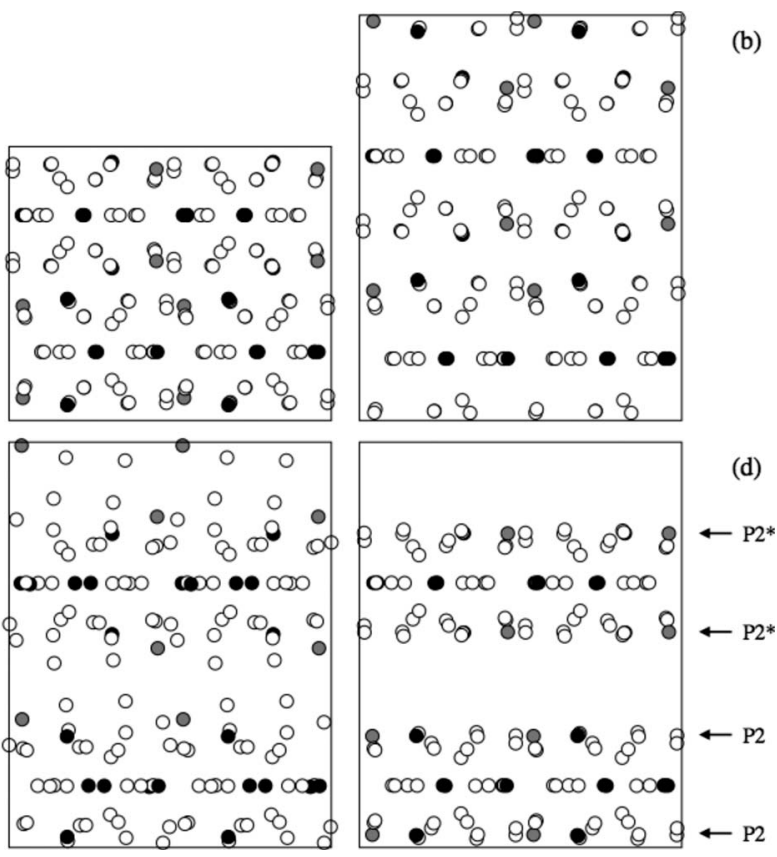

FIG. 6. Determination of the position of the cleavage plane from an $a b$ initio simulation. Here, the mixed Pd-Al sites are replaced by $\mathrm{Pd}$ atoms (gray) while the mixed $\mathrm{Mn}-\mathrm{Al}$ sites are all replaced by $\mathrm{Al}$ atoms (white circles). Mn atoms appear as black spheres. The unit cell of the bulk structure (a) is expanded in the [010] direction (vertical direction) by $50 \%$ (b). After the structural relaxation, the bulk structure has cleaved between the P2 and P2* planes (d). As the unit cell consists of two symmetry-equivalent blocks of atomic planes, two vacuum layers between the blocks are formed. Panel (c) shows an intermediate state when the structure begins to cleave.

inside the dark stars. In the structural model, these two sites labeled TM(5) are occupied by a Mn- $\mathrm{Al}(0.15 / 0.85)$ mixture. Upon annealing, preferential desorptions of $\mathrm{Al} / \mathrm{Mn}$ atoms decorating the $\operatorname{TM}(5)$ sites could explain the formation of dark stars observed on both images of Fig. 5. Similarly, the chemical disorder associated with the TM(5) site could partially explain the bright and diffuse features obtained inside the white pentagons, hence limiting the resolution of the pentagonal chains. Evidently, a contribution in the contrast variation from the surrounding sites, which possess also some substitutional disorder, cannot be excluded.

\section{B. $A b$ initio determination of cleavage plane}

Regarding crystals with complex atomic structures, there is always a question where is the position of the cleavage plane, which atomic plane appears at the surface. In this work based on a comparison of the experimentally observed STM patterns with the structure of individual atomic planes, see Sec. V A, it was concluded that the surface planes of the $\mathrm{T}_{-} \mathrm{Al}_{3}(\mathrm{Mn}, \mathrm{Pd})$ samples are the $\mathrm{P} 2$ planes. Recently, Steurer ${ }^{35}$ proposed a method for the determination of the position of the cleavage plane from an $a b$ initio simulation. The method is demonstrated in Fig. 6. In a model of the bulk structure in the direction [010], the lattice parameter $b$ is expanded by $50 \%$. This leads to a tensile stress in the unit cell larger than the ideal strength of the structure. Figure 6 shows that after a

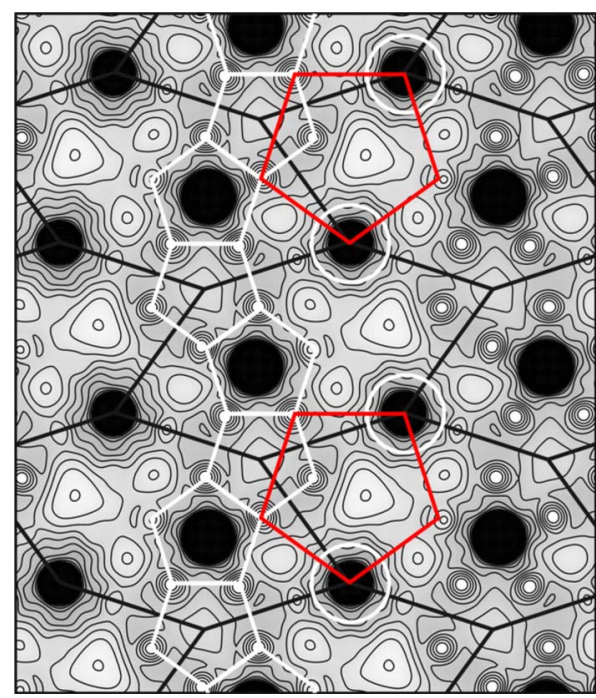

FIG. 7. (Color online) The valence charge-density distribution at the surface P2 plane in the area $25.19 \times 29.43 \AA^{2}$ corresponding to $2 \times 2$ unit cells. The global structure is represented by a tiling of squashed hexagons $\mathrm{H}$ (black lines). The pentagons of $\mathrm{Al}$ atoms are arranged in chains (white pentagons). Between two neighboring Mn atoms (marked by white circles) decorating the vertices of the $\mathrm{H}$ tiles there is a large charge-density depression. Its position is approximately marked by a big (red, gray) pentagon.

structural relaxation, the bulk structure has cleaved between the P2 and $\mathrm{P} 2^{*}$ planes. As the unit cell consists of two symmetry-equivalent blocks of atomic planes two vacuum layers between the blocks are formed. This simulation thus confirms the conclusion deduced from the experiment that the $\mathrm{T}-\mathrm{Al}_{3}(\mathrm{Mn}, \mathrm{Pd})$ crystal cleaves between the $\mathrm{P} 2$ and $\mathrm{P} 2 *$ planes. At this stage, it has to be mentioned that the width of the gap between $\mathrm{P} 2$ and $\mathrm{P} 2{ }^{*}$ planes is the largest interlayer spacing present in the bulk structure.

\section{STM images from $a b$ initio calculations}

For understanding the structure of the surface and the simulated STM images, it is very helpful to look first at the valence charge-density distribution at the surface. Figure 7 shows the charge-density distribution at the surface $\mathrm{P} 2$ plane in the area of $2 \times 2$ unit cells. The global structure is represented by a tiling of squashed hexagons $\mathrm{H}$. Three vertices of the same, say even, parity are occupied by Mn atoms. At the other three vertices of odd parity, there are hollow sites surrounded by a pentagon of $\mathrm{Al}$ atoms. Inside the $\mathrm{H}$ tile one can see a Pd atom surrounded by the same pentagon of $\mathrm{Al}$ atoms but with opposite orientation. The $\mathrm{Al}$ pentagons are arranged in chains. The picture of the charge-density distribution reveals one remarkable structural feature that is not immediately obvious from structural model of the surface. Between two neighboring $\mathrm{Mn}$ atoms decorating the vertices of the $\mathrm{H}$ tiles there is a large charge-density depression. Its position is approximately marked by a big pentagon. While the chains of small pentagons and the chains of Mn atoms are arranged in the characteristic zigzag pattern, these charge-density minima are aligned with much smaller deviations from a straight line. In the simulated STM images these large 


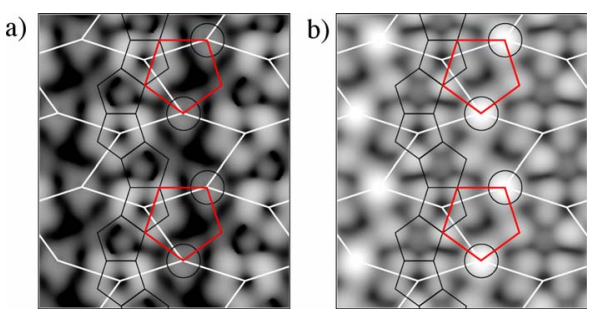

FIG. 8. (Color online) Simulated STM images of the surface P2 plane. Images of the surfaces (a) and (b) are simulated for the bias voltages $-0.4 \mathrm{~V}$ and $+0.4 \mathrm{~V}$, respectively. The area corresponds to $2 \times 2$ unit cells. The chains of the small pentagons (black), big pentagons (red, gray), circles (black), and the tiling of squashed hexagons represent the structural features of the surface described in Fig. 7. The bias dependency of the images observed experimentally is also seen on the simulated images.

charge-density depressions are seen as dark spots.

Figure 8 presents simulated STM images of the same model of the P2 surface. The images are calculated for a constant tunneling current and the bias voltages of $V_{\text {bias }}=-0.4$ and $+0.4 \mathrm{~V}$ corresponding to the occupied and unoccupied states around the Fermi level, respectively. The bias dependency of the images observed experimentally is clearly seen also on the simulated images. The contrast inside the big pentagon is substantially changed. The position and the darkness of the spot inside the pentagon varies with the bias voltage. Large variations in the contrast with the bias are observed also at the $\mathrm{Mn}$ sites and near the center of the $\mathrm{H}$ tiles. The bright spots corresponding to the $\mathrm{Al}$ pentagons around the odd vertices of the $\mathrm{H}$ tiles can be well recognized at both bias voltages. The simulated images qualitatively reproduce characteristic features observed in the experimental images. However, because of the chemical disorder inherent to the real structure and because only one chemical variant has been considered in our calculations, a one-to-one comparison between the simulated and experimental STM images would be meaningless.

\section{Surface investigated by XPD measurements}

The same surface has been further analyzed using x-ray photoelectron diffraction. The measurements have been carried out after annealing the $\mathrm{T}-\mathrm{Al}_{3}(\mathrm{Mn}, \mathrm{Pd})$ crystal to $873 \mathrm{~K}$ for $1 \mathrm{~h}$. Figures 9(a), 9(c), and 9(e) exhibit the $\mathrm{Al} 2 s$, $\operatorname{Pd} 3 d_{5 / 2}$, and $\operatorname{Mn} 2 p_{3 / 2}$ photoemission line intensity for different angular and polar emission angles. Normal emission $\left(0^{\circ}\right)$ corresponds to the central spot of the XPD pattern while the outer ring represents an emission angle of $70^{\circ}$. The bright and dark contrasts on these stereographic projections indicate high and low intensities, respectively. The XPD patterns measured for selected emitters ( $\mathrm{Al} 2 s, \quad \mathrm{Pd} 3 d_{5 / 2}$, and $\mathrm{Mn} 2 p_{3 / 2}$ ) using $\mathrm{Al} K \alpha$ radiation (1486.7 eV) reveal typical features of decagonal symmetry elements. Indeed, several rings of ten equivalent intense spots and a central 10f symmetry axis dominate all XPD patterns. The patterns presented in Figs. 9(a), 9(c), and 9(e) are similar to those observed on the $d$-Al-Ni-Co, ${ }^{36}$ on the decagonal quasicrystal overlayer grown on the fivefold surface of the $i$-Al-Pd-Mn

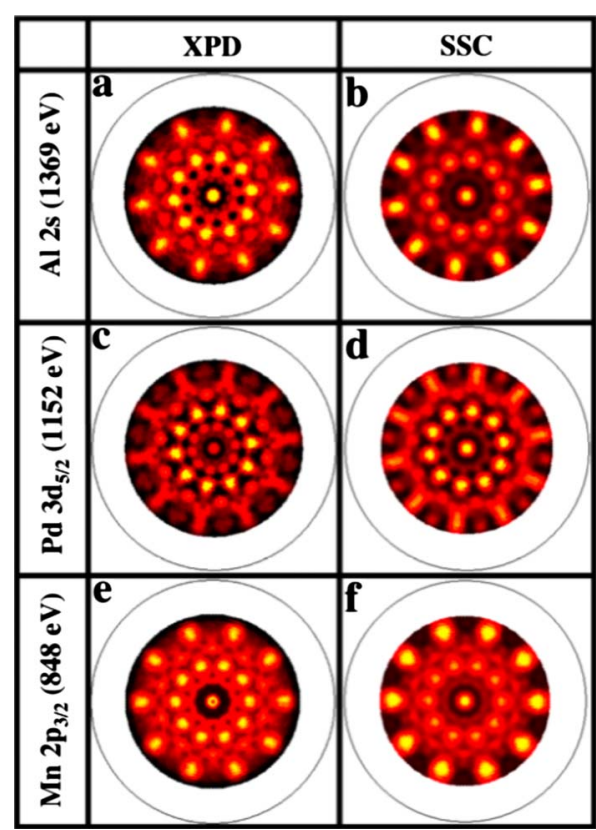

FIG. 9. (Color online) Experimental XPD patterns of (a) Al $2 s$, (c) $\mathrm{Pd} 3 d_{5 / 2}$, and (e) $\mathrm{Mn} 2 p_{3 / 2}$ core levels measured on the $\mathrm{T}-\mathrm{Al}_{3}(\mathrm{Mn}, \mathrm{Pd})(010)$ surface with $\mathrm{Al} K \alpha(1486.7 \mathrm{eV})$ x-ray source. Single scattering cluster simulations for (b) Al $2 s\left(E_{\text {Kin }}=1369 \mathrm{eV}\right)$, (d) $\operatorname{Pd} 3 d_{5 / 2}\left(E_{K i n}=1152 \mathrm{eV}\right)$, and (f) $\operatorname{Mn} 2 p_{3 / 2}\left(E_{K i n}=848 \mathrm{eV}\right)$ emissions based on a cluster derived from the bulk model (Ref. 17) described in Fig. 1.

sample ${ }^{37}$ and recently on the $\mathrm{Al}_{13} \mathrm{Co}_{4}$ crystal. ${ }^{32}$ As XPD probes the local real-space environnement around the selected emitting atoms, these measurements reveal an average short-range decagonal ordering around the $\mathrm{Al}, \mathrm{Pd}$, and $\mathrm{Mn}$ atoms at the near surface. To interpret the experimental diffractograms, single scattering cluster (SSC) simulations for the $\mathrm{Al} 2 s, \mathrm{Pd} 3 d_{5 / 2}$, and $\mathrm{Mn} 2 p_{3 / 2}$ emissions have been performed based on the available $\mathrm{T}-\mathrm{Al}_{3}(\mathrm{Mn}, \mathrm{Pd})$ structural bulk model. ${ }^{17}$ The model used for the SSC simulations contains 1248 atoms (eight unit cells), 20 atomic plans along the [010] direction and has the following dimensions: 29.434 $\times 25.020 \times 25.188 \AA^{3}$. Five separate clusters have been generated to represent the different atomic configurations that can be observed at the surface. Hence, the surface of the five models is either terminated by the two layers F-P1 (F layer as topmost termination, $\mathrm{P} 1$ is the layer below the $\mathrm{F}$ layer), P1-F, P2-P2, P1-P2, or by P2-P1. The cluster used to carry out the calculations consists of 1248 atoms with $107 \mathrm{Al}, 11$ $\mathrm{Pd}$, and $44 \mathrm{Mn}$ as emitters. The photoelectron intensity maps obtained for each emitter appear similar regardless of the model chosen. The SSC patterns presented in Fig. 9 have been obtained for the P2-P1 configuration. The experimental XPD patterns for the $\mathrm{Al} 2 s, \mathrm{Pd} 3 d$, and $\mathrm{Mn} 2 p$ can be nicely reproduced by the SSC simulations [Figs. 9(b), 9(d), and $9(\mathrm{f})]$ which display the expected decagonal rings made of ten distinct spots. The patterns are well reproduced in terms of positions but differ slightly in the intensity distribution. However, the intensity distributions are well reproduced, for instance, for the Mn emitter with the external decagonal ring situated at $\theta=62^{\circ}$ [Fig. 9(f)]. The position and intensity in 


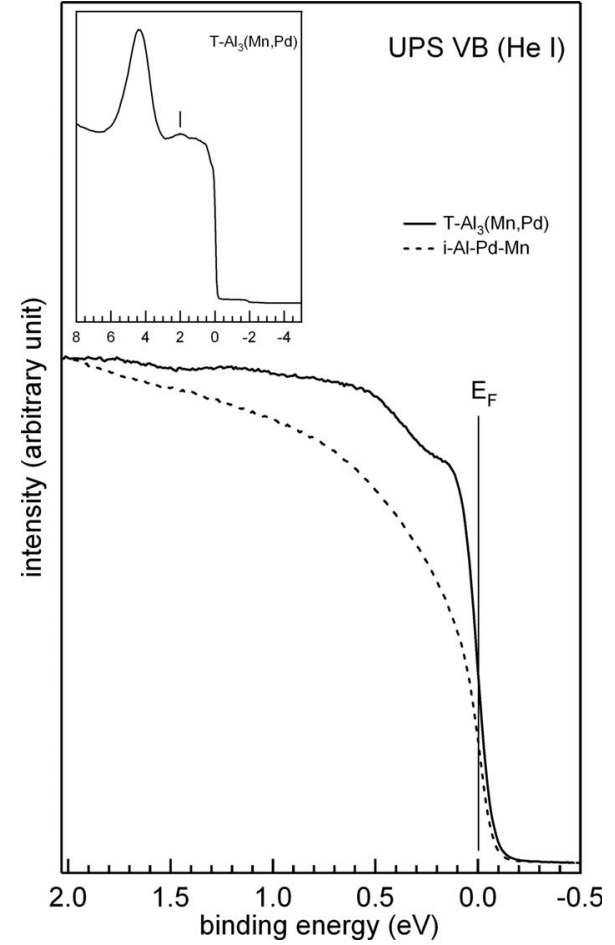

FIG. 10. Near $E_{F}$ UPS valence-band spectra of T- $\mathrm{Al}_{3}(\mathrm{Mn}, \mathrm{Pd})$ (solid line) and $i$-Al-Pd-Mn (dashed line). The position of $E_{F}$ is indicated by a dashed line. Spectra are normalized to have the same height at $2 \mathrm{eV}$ binding energy. Inset: valence-band spectrum of the $\mathrm{T}_{-} \mathrm{Al}_{3}(\mathrm{Mn}, \mathrm{Pd})$ up to $8 \mathrm{eV}$. The main peak at $4.5 \mathrm{eV}$ corresponds to $\mathrm{Pd} 4 d$ states and the vertical line is located at $2 \mathrm{eV}$.

the two decagonal rings situated on $\theta=36^{\circ}$ and $48^{\circ}$ are accurately reproduced for the $\mathrm{Pd}$ emitter too. While a relatively good agreement is obtained between XPD maps and SSC images, it is not possible to discriminate between the most preferred topmost surface termination. However, it clearly appears that the $p$-10f symmetry of the bulk structure is preserved within the near-surface region.

\section{E. Electronic character of the surface}

We now turn into the characterization of the $\mathrm{T}-\mathrm{Al}_{3}(\mathrm{Mn}, \mathrm{Pd})$ electronic structure using XPS and UPS techniques. The photoemission spectra are compared to those obtained on the icosahedral Al-Pd-Mn quasicrystal. The UPS valence-band (VB) spectra in the near $E_{F}$ region collected on both the $i$-Al-Pd-Mn quasicrystal (dashed line) and on the $\mathrm{T}-\mathrm{Al}_{3}(\mathrm{Mn}, \mathrm{Pd})$ sample (solid line) are presented in Fig. 10. The valence-band spectrum of the Taylor phase up to $8 \mathrm{eV}$ is presented in the inset where the main peak around $4.5 \mathrm{eV}$ corresponds to $\mathrm{Pd} 4 d$ states. It is known that $\mathrm{Mn} d$ states dominate the density of states (DOS) around $E_{F}$ in $i$-Al-Pd-Mn and Al-Mn alloys. The rounded shape of the spectral intensity near $E_{F}$ has been considered as the signature of the pseudogap, i.e., a reduction in the DOS at the Fermi level. ${ }^{9,24,38-40}$ From the comparison of two spectra normalized at $2 \mathrm{eV}$, the Fermi edge of $\mathrm{T}-\mathrm{Al}_{3}(\mathrm{Mn}, \mathrm{Pd})$ appears much sharper than the one observed on the $i$-Al-Pd-Mn quasicrystal. This is partly explained by an enhanced Mn $d$ DOS

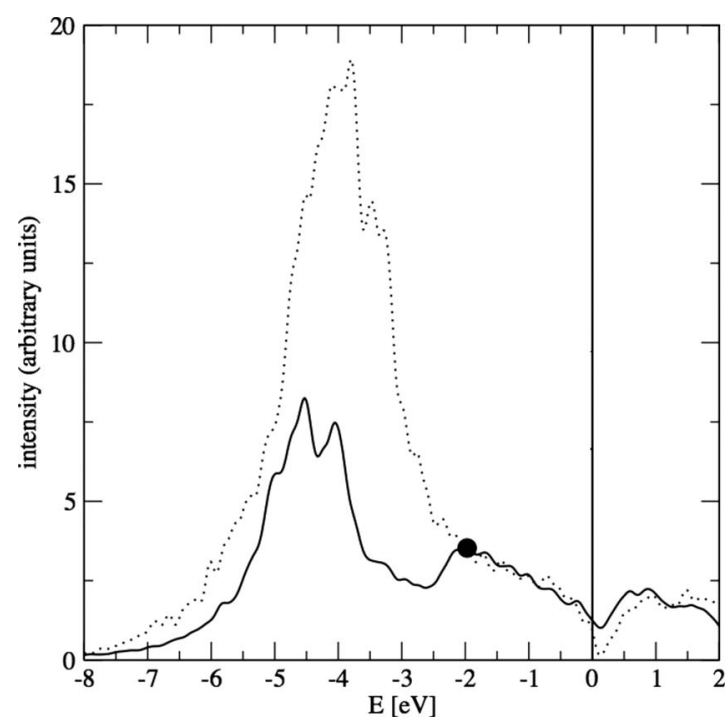

FIG. 11. Calculated UPS VB (He I) spectrum for the bulk of $\mathrm{T}-\mathrm{Al}_{3}(\mathrm{Mn}, \mathrm{Pd})$ (full line) and $i$-Al-Pd-Mn (dashed line) samples. The spectra are normalized to have the same height at the energy of $-2 \mathrm{eV}$ indicated by a black dot.

at $E_{F}$ due to a higher concentration of $\mathrm{Mn}$ in $\mathrm{T}-\mathrm{Al}_{3}(\mathrm{Mn}, \mathrm{Pd})$ crystal than in the $i$-Al-Pd-Mn sample. Nevertheless, the decrease in the spectral intensity between the Fermi edge and $0.5 \mathrm{eV}$ below $E_{F}$ could be interpreted as an evidence for a shallow pseudogap in the DOS of the $\mathrm{T}-\mathrm{Al}_{3}(\mathrm{Mn}, \mathrm{Pd})$ phase. However, the procedure introduced by Mori et al. ${ }^{41}$ to quantify the depth and width of the pseudogap measured on the icosahedral quasicrystal cannot be applied here as the DOS near $E_{F}$ cannot be considered as a smooth function of the energy. From a qualitative perspective, the presence of the pseudogap is not evident in the $\mathrm{T}-\mathrm{Al}_{3}(\mathrm{Mn}, \mathrm{Pd}) \mathrm{VB}$ spectrum and the T-phase sample exhibits a more metallic character than its quasicrystal counterpart.

The UPS valence-band spectra of the $\mathrm{T}-\mathrm{Al}_{3}(\mathrm{Mn}, \mathrm{Pd})$ and $i$-Al-Pd-Mn samples have been calculated using the bulk DOS. The Katz-Gratias-Boudard model is used in our calculations of the $i$-Al-Pd-Mn properties. ${ }^{9,42}$ This is justified as most of the UPS signal comes from the near-surface region for He I radiation $(21.2 \mathrm{eV})$. The UPS spectrum is approximated by summing the orbital contributions of the DOS of $\mathrm{Al}, \mathrm{Mn}$, and $\mathrm{Pd}$ weighted by the corresponding crosssections for He I energies. The experimental broadening is not taken into account in the calculations. As shown in Fig. 11 , the spectra have been normalized to have the same height at the energy of $2 \mathrm{eV}$ as per Fig. 10. The calculated UPS VB spectra exhibit a very deep pseudogap at $E_{F}$ for the $i$-Al-Pd-Mn quasicrystal but a much shallower pseudogap for the $\mathrm{T}-\mathrm{Al}_{3}(\mathrm{Mn}, \mathrm{Pd})$ crystal. This is in agreement with both spectra presented in Fig. 10. The position of the main features (Pd $4 d$ states, for instance) are also comparable between the large scale UPS spectra (inset of Fig. 10) and the calculated curve (see Fig. 11). The less intense Mn $3 d$ peak at $2 \mathrm{eV}$ below $E_{F}$ on the experimental UPS spectra compared to the calculated curve could be explained by the atomic decoration used for the DOS calculation and/or by a loss of resolution due to instrumental broadening. 
An alternative, although indirect, approach to assess the DOS at $E_{F}$ is to study the asymmetry of the Mn $2 p$ core-level line shape. Line shape of the Mn $2 p$ core level has been well studied for $i$-Al-Pd-Mn, Al-Mn alloys, and Mn metal using Doniach-Šunjić (DS) function. ${ }^{23,24,40,43,44}$ Metallic core levels are asymmetric because of electron-hole pair excitation across $E_{F}$ and the DS asymmetry parameter $(\alpha)$ is given by ${ }^{45}$

$$
\sum_{q<2 q_{F}} \frac{\left|V_{Q}\right|^{2}}{|\epsilon(q, 0)|^{2}} \frac{N(0)}{q V_{F}}
$$

where $N(0)$ is the DOS at $E_{F}, V_{Q}$ is the core-hole potential, $q$ is the momentum vector, $V_{F}$ is the Fermi velocity, and $\epsilon$ is the dielectric function. Thus, the estimation of the asymmetry parameter $\alpha$ is an indirect probe of the DOS at $E_{F} N(0)$. It should be noted that the DS asymmetry parameter $(\alpha)$ for $i$-Al-Pd-Mn is significantly lower than that of Mn metal. This has been related to the low $N(0)$ in the $i$-Al-Pd-Mn due to the presence of the pseudogap. ${ }^{23}$ The $\alpha$ parameter of the Mn $2 p$ core level was found to be 0.46 for $\mathrm{Mn}$ metal and it turned out to be as low as $0.14-0.18$ for $i$-Al-Pd-Mn. Recently, it has been shown that the value of $\alpha$ of Mn $2 p$ for Mn metal has been over estimated due to the lack of consideration of the $1 \mathrm{eV}$ satellite while simulating the core-level line shape. Indeed, the Mn $2 p$ core level of bulklike Mn film has been studied using high-resolution photoemission spectroscopy and an unusual satellite feature at $1 \mathrm{eV}$ higher binding energy (BE) from the main peak has been observed. ${ }^{44}$ It originates from the intra-atomic multiplet effect related to $\mathrm{Mn}$ atoms with large local moment. ${ }^{44}$ Taking into account this satellite in pure Mn leads to a lower value of the $\alpha$ parameter which is then equal to $0.34 .{ }^{44}$ This satellite feature was not observed in the Mn $2 p$ core level of $i$-Al-Pd-Mn and Al-Mn alloys and only one DS function was sufficient to simulate the Mn $2 p$ core level for such systems. ${ }^{23,24,40,44}$ Strong Al $3 s$, $p$-Mn $3 d$ hybridization was suggested to be responsible for the suppression of $1 \mathrm{eV}$ satellite feature in $i$-Al-Pd-Mn and Al-Mn alloys. ${ }^{40,44}$

Figure 12 shows the Mn 2p $p_{3 / 2}$ core level spectrum (a) for the $\mathrm{T}-\mathrm{Al}_{3}(\mathrm{Mn}, \mathrm{Pd})$ and (b) for the $i$-Al-Pd-Mn samples. Both the spectra have been recorded with similar analyzer settings and thus with similar energy resolution. Visual inspection of the two spectra shows that the core-level line shape is more asymmetric toward the higher $\mathrm{BE}$ side for $\mathrm{T}-\mathrm{Al}_{3}(\mathrm{Mn}, \mathrm{Pd})$ compared to $i$-Al-Pd-Mn. We have performed a least-square fitting to simulate the $\mathrm{Mn} 2 p_{3 / 2}$ line shape of $\mathrm{T}-\mathrm{Al}_{3}(\mathrm{Mn}, \mathrm{Pd})$ and $i$-Al-Pd-Mn using a DS function. The intrinsic lifetime broadening of the core level $(2 \gamma), \alpha$, intensity and position of core level, and the iterative background are varied independently during the fitting. The residuals in Figs. 12(a) and 12(b) show the good quality of the fit. For $i$-Al-Pd-Mn, we obtained a value $\alpha=0.140 \pm 0.015$ while $2 \gamma$ turned out to be $0.32 \pm 0.02$. These values are in good agreement with the values obtained from the DS line shape simulation of highresolution $\mathrm{Mn} 2 p$ core level of $i$-Al-Pd-Mn reported in the literature. $^{22,24}$ DS line shape simulation of $\mathrm{Mn} 2 p_{3 / 2}$ line for $\mathrm{T}-\mathrm{Al}_{3}(\mathrm{Mn}, \mathrm{Pd})$ resulted in $\alpha(=0.200 \pm 0.013)$ while $2 \gamma$ was equal to $0.36 \pm 0.01$. This is consistent with a shallower

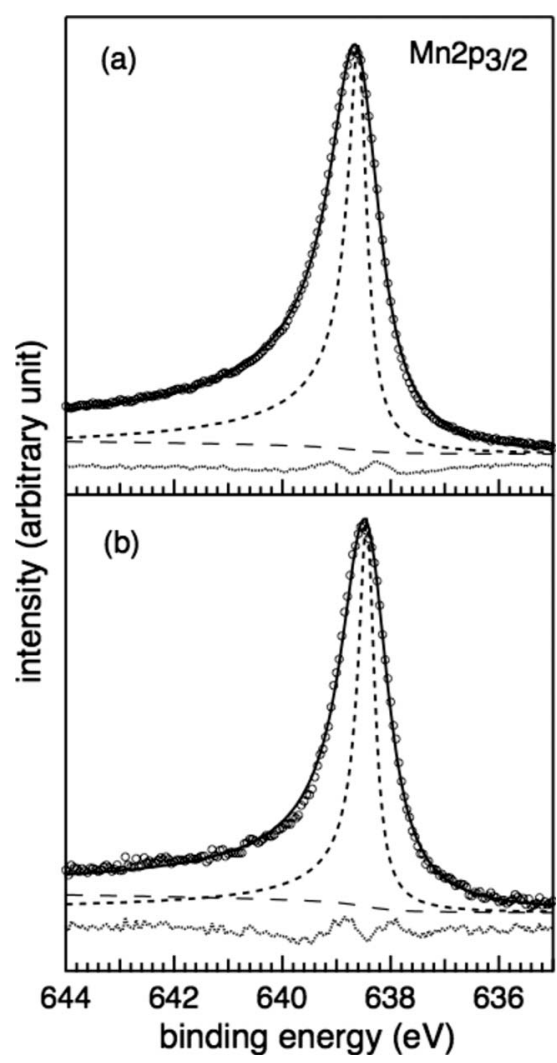

FIG. 12. Mn 2p $p_{3 / 2}$ core-level spectra (open circles) for (a) $\mathrm{T}-\mathrm{Al}_{3}(\mathrm{Mn}, \mathrm{Pd})$ and (b) $i$-Al-Pd-Mn. The fitted spectra (thin solid line), the spectra deconvoluted from experimental broadening (short dashed line), the iterative background (long dashed line) and the residual (dots) are also shown.

pseudogap in the $\mathrm{T}-\mathrm{Al}_{3}(\mathrm{Mn}, \mathrm{Pd})$ phase compared to the $i$-Al-Pd-Mn sample due to an enhanced Mn $d$ contribution to the DOS near $E_{F}$.

\section{DISCUSSION}

The analysis of the (010) surface of $\mathrm{T}-\mathrm{Al}_{3}(\mathrm{Mn}, \mathrm{Pd})$ indicates that there is no evident lateral-surface reconstruction but a high degree of structural imperfections. While some of the contrast disparities on STM images are expected due to the chemical disorder intrinsic to the bulk model, hollow sites resembling "dark" stars have been observed within the topmost surface layer. These surface vacancies exhibit similar sizes as those encountered on the fivefold Al-Pd-Mn quasicrystal surface, then attributed to the irregular structure of the first shell of the pseudo-Mackay cluster. ${ }^{46}$ On the $\mathrm{T}-\mathrm{Al}_{3}(\mathrm{Mn}, \mathrm{Pd})$ surface, the origin of these depressions, which are not present in the structural model, have been tentatively attributed to missing atoms at sites referred as $\operatorname{TM}(5)$ by Klein et al. ${ }^{17}$ The latter atomic positions are supposedly occupied by a mixture of $\mathrm{Mn} / \mathrm{Al}$ atoms. In fact, when populated, this site contributes significantly to the intensity of the STM image [see Fig. 5(b)]. Atomic desorptions have been recently reported by Addou et $a .^{32}$ when investigating the $\mathrm{Al}_{13} \mathrm{Co}_{4}(100)$ approximant surface. In that case, only ten out of $20 \mathrm{Al}$ atoms per surface unit cell are preserved within the 
topmost plane. This selective desorption was interpreted by a particular bonding present within the 23-atom bipentagonal pyramid clusters. ${ }^{47,48}$ Here, the $\mathrm{TM}(5)$ site belongs to interpenetrating icosahedral clusters that form the column, basic structural constituent, presented in Fig. 1(d). While information about the intercluster bonding along the [010] direction remain scarce, ${ }^{8}$ the erratic occupation of $\mathrm{TM}(5)$ sites could be explained by the decoration of the in-plane nearestneighboring sites, namely, $\mathrm{TM}(1)$ and $\mathrm{TM}(3)$. As mentioned previously, both sites also exhibit a substitutional disorder and can be populated by either $\mathrm{Al}$ or $\mathrm{Mn}$ atoms. Depending on the elements present at $\operatorname{TM}(1)$ and $\operatorname{TM}(3)$ sites, the bonding with $\mathrm{Mn} 0.15-\mathrm{Al} 0.85$ atoms at $\mathrm{TM}(5)$ site should be considerably different. Hence, it is reasonable to assume that stronger or weaker (attractive/repulsive) interactions between atoms at $\mathrm{TM}(5)$ positions with the surrounding will result. In addition, the reduced coordination of $\mathrm{Mn} / \mathrm{Al}$ atoms at $\mathrm{TM}(5)$ site at the surface could favor desorption.

The structural analysis of atomically resolved STM images and a qualitative comparison with simulated images indicate that the surface is terminated by a P2-like plane present in the bulk model. The step height measurements equal to $b / 2$ are in agreement with only one type of surface termination, with the exception of the limited regions at the edges of the terraces. Step heights values consistent with half of the lattice parameter have also been measured on other approximant surfaces. ${ }^{13,32,49}$ With P1 lying theoretically at $0.625 \AA$ beneath $\mathrm{P} 2$, the combined planes lead to a denser surface than if a flat layer would be selected as the surface termination. Statistically, the puckered surface $(\mathrm{P} 2+\mathrm{P} 1)$ should also be Al rich compared to the flat layer. The surface termination is then created by a cut through the bulk model at the widest interlayer gap along the [010] direction. All these results are consistent with previous studies performed on approximant and quasicrystal surfaces where the exposed surface layers correspond to a cleavage through the bulk model at largest gaps. ${ }^{50}$ The (pseudo) fivefold and (pseudo) tenfold surface planes have been also reported to be laterally bulk terminated. ${ }^{15,16,46,51,52}$ It has to be mentioned that the $\xi^{\prime}$-Al-Pd-Mn (010) and the $\mathrm{Al}_{13} \mathrm{Co}_{4}$ (100) topmost surfaces correspond to incomplete bulk planes. ${ }^{13,32}$ For Al-based quasicrystals like the fivefold $i$-Al-Pd-Mn surface, the average density of the two terminating planes ${ }^{15}$ and the $\mathrm{Al}$ concentration are high. ${ }^{46}$ Out of the two topmost planes (only $0.38 \AA$ apart ${ }^{51}$ ), the layer having the highest concentration of $\mathrm{Al}$ atoms is exposed at the surface. This plane selection is not verified for the $\mathrm{T}-\mathrm{Al}_{3}(\mathrm{Mn}, \mathrm{Pd})(010)$ surface. If $\mathrm{P} 2$ and $\mathrm{P} 1$ layers are combined to form a single termination $\mathrm{P}$, they form the densest Al-rich surface plane compared to the F termination. However, the layer containing TM elements (mainly Mn atoms) is this time favored as the topmost surface layer. As explained above, the cleavage plane necessary to generate the surface layers is always positioned in between consecutive puckered $\mathrm{P}(\mathrm{P} 2+\mathrm{P} 1)$ layers. Due to their Al-rich/pure content, the bonding between $\mathrm{P} 1$ and $\mathrm{F}$ layers can be considered in a first approximation as originating from an $\mathrm{Al}-\mathrm{Al}$ atomic interaction. The bonding between two TM-rich P2 layers should be considerably different and may influence the cut height through the bulk model. As confirmed by DFT calculations (see Fig. 6), the cleavage plane is indeed in be- tween P2 and P2* layers. From the surface structural imperfections and the chemical disorder inherent to the bulk model, it is not possible to estimate the overall TM concentration at the extreme surface. However, the results obtained using XPS $\left(\mathrm{Al}_{75.4} \mathrm{Pd}_{3.3} \mathrm{Mn}_{21.3}\right.$ with an error of $\left.\pm 10 \%\right)$ show that there is no chemical segregation at the surface of the $\mathrm{T}-\mathrm{Al}_{3}(\mathrm{Mn}, \mathrm{Pd})$ crystal.

In addition using LEED and XPD techniques, it has been shown that the pseudotenfold symmetry is clearly maintained at the sample surface. The LEED patterns exhibit sets of decagonal rings, which are more distinguishable here than on the LEED patterns recorded on the $p$-10f surface of the orthorhombic $\mathrm{Al}_{13} \mathrm{Co}_{4}$ approximant. However, it remains less apparent than on the $\xi^{\prime}-\mathrm{Al}-\mathrm{Pd}-\mathrm{Mn}$ sample. ${ }^{13}$ In both cases, the surface planes have been related to layers present in the bulk models. The difference in the diffraction patterns can be explained by denser planes at the $\mathrm{T}-\mathrm{Al}_{3}(\mathrm{Mn}, \mathrm{Pd})$ surface than on the $\mathrm{Al}_{13} \mathrm{Co}_{4}$ approximant, where the surface terminations consist of incomplete puckered layers. ${ }^{32}$ For both approximants, SSC calculations reproduce the local decagonal symmetry expected at the near-surface region around each emitter but does not allow to discriminate between preferred surface terminations.

It has been demonstrated that a structure-induced pseudogap in the $\mathrm{Al}$ band exists in the decagonal $\mathrm{Al}_{70} \mathrm{Pd}_{13} \mathrm{Mn}_{17}$ sample and its approximant phases. ${ }^{8,53}$ The $s p-d$ hybridization plays a crucial role in the formation of the pseudogap. ${ }^{54}$ In the case of the $\mathrm{T}-\mathrm{Al}_{3} \mathrm{Mn}$ phase and compared to decagonal quasicrystal, the high concentration of Mn atoms should broaden the Mn band which would then overlap with the Fermi level, hence leading to a higher DOS at $E_{F}$. The high concentration and the chemical order of $\mathrm{Mn}$ atoms should lead to a bonding-antibonding splitting characteristic of transition-metal $d$ bands, due to the loss of the impurity character of the $d$ band. ${ }^{8}$ As shown in Fig. 10, a higher spectral intensity is also observed for the $\mathrm{T}-\mathrm{Al}_{3}(\mathrm{Mn}, \mathrm{Pd})$ phase compared to the quasicrystal sample. In addition, several components which can be related to the Mn $d$ band have been observed near $E_{F}$. From an additional Mn $2 p$ core-level analysis, it appears that the $\mathrm{T}-\mathrm{Al}_{3}(\mathrm{Mn}, \mathrm{Pd})$ surface presents a more metallic character than the fivefold $i$-Al-Pd-Mn quasicrystal surface, i.e., a pseudogap of reduced depth.

\section{CONCLUSIONS}

We have investigated the (010) surface of the recently grown orthorhombic $\mathrm{T}-\mathrm{Al}_{3}(\mathrm{Mn}, \mathrm{Pd})$ crystal. The LEED patterns and the XPD diffractograms show the expected pseudotenfold symmetry of the surface. Upon annealing the sample to $923 \mathrm{~K}$, we observe atomically flat terraces separated mainly by a single-step height equal to one half of the unit-cell parameter $(b / 2)$. Despite the presence of structural imperfections, the surface termination has been related to the puckered layer present in the structural bulk model. From the STM analysis and the $a b$ initio simulation of the cleavage process, we conclude that the surface topmost layer consists of the P2 plane (containing transition-metal elements) with the $\mathrm{P} 1$ plane (Al rich) $0.65 \AA$ beneath. The drastic bias de- 
pendency observed on experimental images is also verified on simulated STM images calculated from the P2 layer. From UPS and XPS measurements, we have shown that this Taylor phase exhibits a higher density of states at the Fermi level compared to the $i$-Al-Pd-Mn quasicrystal.

\section{ACKNOWLEDGMENTS}

The European network of Excellence on "Complex Metallic Alloys," Contract No. NMP3-CT-2005-500145, and the
Agence Nationale de la Recherche, Reference No. ANR-07Blan-0270, are acknowledged for their financial support. The work has been also supported by the Austrian Ministry for Education, Science and Art through the Center for Computational Materials Science. M.K. thanks also for support from the Grant Agency for Science of Slovakia (Grant No. 2/5096/ $25)$ and from the Slovak Research and Development Agency (Grant No. APVV-0413-06, CEX-Nanosmart). S. R. Barman is thanked for the use of the XPS fitting procedure.
*Present address: Fritz-Haber-Institut der Max-Planck-Gesellschaft Abteilung Molekulphysik, Faradayweg 4-6, 14195 Berlin, Germany.

${ }^{\dagger}$ Corresponding author; ledieu@1sg2m.org

${ }^{1}$ Basics of Thermodynamics and Phase Transitions in Complex Intermetallics, edited by E. Belin-Ferré (World Scientific, Singapore, 2008).

${ }^{2}$ M. Audier, M. Durand-Charre, and M. De Boissieu, Philos. Mag. B 68, 607 (1993)

${ }^{3}$ M. A. Taylor, Acta Crystallogr. 14, 84 (1961).

${ }^{4}$ K. Robinson, Acta Crystallogr. 7, 494 (1954).

${ }^{5}$ S. Balanetskyy, G. Meisterernst, M. Heggen, and M. Feuerbacher, Intermetallics 16, 71 (2008).

${ }^{6}$ K. Hiraga, M. Kaneko, Y. Matsuo, and S. Hashimoto, Philos. Mag. B 67, 193 (1993).

${ }^{7}$ X. Z. Li and K. H. Kuo, Philos. Mag. B 65, 525 (1992).

${ }^{8}$ M. Krajčí, J. Hafner, and M. Mihalkovič, Phys. Rev. B 55, 843 (1997).

${ }^{9}$ M. Krajčí, M. Windisch, J. Hafner, G. Kresse, and M. Mihalkovič, Phys. Rev. B 51, 17355 (1995).

${ }^{10}$ M. Krajčí and J. Hafner, Phys. Rev. B 71, 054202 (2005).

${ }^{11}$ H. R. Sharma, M. Shimoda, and A. P. Tsai, Adv. Phys. 56, 403 (2007).

${ }^{12}$ J. Ledieu, C. A. Muryn, G. Thornton, G. Cappello, J. Chevrier, R. D. Diehl, T. A. Lograsso, D. Delaney, and R. McGrath, Mater. Sci. Eng., A 294-296, 871 (2000).

${ }^{13}$ V. Fournée, A. R. Ross, T. A. Lograsso, J. W. Anderegg, C. Dong, M. Kramer, I. R. Fisher, P. C. Canfield, and P. A. Thiel, Phys. Rev. B 66, 165423 (2002).

${ }^{14}$ M. Boudard, H. Klein, M. de Boissieu, M. Audier, and H. Vincent, Philos. Mag. A 74, 939 (1996).

${ }^{15}$ Z. Papadopolos, G. Kasner, J. Ledieu, E. J. Cox, N. V. Richardson, Q. Chen, R. D. Diehl, T. A. Lograsso, A. R. Ross, and R. McGrath, Phys. Rev. B 66, 184207 (2002).

${ }^{16}$ L. Barbier, D. Le Floc'h, Y. Calvayrac, and D. Gratias, Phys. Rev. Lett. 88, 085506 (2002).

${ }^{17}$ H. Klein, M. Boudard, M. Audier, M. de Boissieu, H. Vincent, L. Beraha, and M. Duneau, Philos. Mag. Lett. 75, 197 (1997).

${ }^{18}$ E. Belin and A. Traverse, J. Phys.: Condens. Matter 3, 2157 (1991).

${ }^{19}$ G. Trambly de Laissardière, D. Nguyen Manh, L. Magaud, J. P. Julien, F. Cyrot-Lackmann, and D. Mayou, Phys. Rev. B 52, 7920 (1995).

${ }^{20}$ J. M. Dubois, Useful Quasicrystals (World Scientific, Singapore, 2005).
${ }^{21}$ G. Neuhold, S. R. Barman, K. Horn, W. Theis, Ph. Ebert, and K. Urban, Phys. Rev. B 58, 734 (1998).

${ }^{22}$ V. Fournée, P. J. Pinhero, J. W. Anderegg, T. A. Lograsso, A. R. Ross, P. C. Canfield, I. R. Fisher, and P. A. Thiel, Phys. Rev. B 62, 14049 (2000).

${ }^{23}$ V. Fournée, J. W. Anderegg, A. R. Ross, T. A. Lograsso, and P. A. Thiel, J. Phys.: Condens. Matter 14, 2691 (2002).

${ }^{24}$ K. Horn, W. Theis, J. J. Paggel, S. R. Barman, E. Rotenberg, Ph. Ebert, and K. Urban, J. Phys.: Condens. Matter 18, 435 (2006).

${ }^{25}$ Y. Matsuo, M. Kaneko, T. Yamanoi, N. Kaji, K. Sugiyama, and K. Hiraga, Philos. Mag. Lett. 76, 357 (1997).

${ }^{26}$ G. Kresse and J. Furthmüller, Phys. Rev. B 54, 11169 (1996).

${ }^{27}$ G. Kresse and D. Joubert, Phys. Rev. B 59, 1758 (1999).

${ }^{28}$ J. P. Perdew and Y. Wang, Phys. Rev. B 45, 13244 (1992).

${ }^{29}$ P. E. Blöchl, Phys. Rev. B 50, 17953 (1994).

${ }^{30}$ J. Tersoff and D. R. Hamann, Phys. Rev. B 31, 805 (1985).

${ }^{31}$ M. Mihalkovič and M. Widom, alloy database at http:// alloy.phys.cmu.edu/

${ }^{32}$ R. Addou, E. Gaudry, Th. Deniozou, M. Heggen, M. Feuerbacher, P. Gille, Yu. Grin, R. Widmer, O. Gröning, V. Fournée, J.-M. Dubois, and J. Ledieu, Phys. Rev. B 80, 014203 (2009).

${ }^{33}$ J. Ledieu, E. J. Cox, R. McGrath, N. V. Richardson, Q. Chen, V. Fournée, T. A. Lograsso, A. R. Ross, K. J. Caspersen, B. Unal, J. W. Evans, and P. A. Thiel, Surf. Sci. 583, 4 (2005).

${ }^{34}$ J. Ledieu and R. McGrath, J. Phys.: Condens. Matter 15, S3113 (2003).

${ }^{35} \mathrm{~W}$. Steurer (private communication).

${ }^{36}$ D. Naumović, P. Aebi, L. Schlapbach, C. Beeli, T. A. Lograsso, and D. W. Delaney, Proceedings of the Sixth International Conference on Quasicrystals, Yamada Conference XLVII, edited by S. Takeuchi and T. Fujiwara (World Scientific, Singapore, 1998).

${ }^{37}$ M. Shimoda, J. Q. Guo, T. J. Sato, and A. P. Tsai, Surf. Sci. 454-456, 11 (2000).

${ }^{38}$ Z. M. Stadnik, D. Purdie, M. Garnier, Y. Baer, A.-P. Tsai, A. Inoue, K. Edagawa, and S. Takeuchi, Phys. Rev. Lett. 77, 1777 (1996).

${ }^{39}$ Z. M. Stadnik, D. Purdie, M. Garnier, Y. Baer, A.-P. Tsai, A. Inoue, K. Edagawa, S. Takeuchi, and K. H. J. Buschow, Phys. Rev. B 55, 10938 (1997).

${ }^{40}$ A. K. Shukla, C. Biswas, R. S. Dhaka, S. C. Das, P. Krüger, and S. R. Barman, Phys. Rev. B 77, 195103 (2008).

${ }^{41}$ M. Mori, S. Matsuo, T. Ishimasa, T. Matsuura, K. Kamiya, H. Inokuchi, and T. Matsukawa, J. Phys.: Condens. Matter 3, 767 (1991).

${ }^{42}$ M. Krajčí and J. Hafner, Phys. Rev. B 80, 214419 (2009). 
${ }^{43}$ C. J. Jenks, S.-L. Chang, J. W. Anderegg, P. A. Thiel, and D. W. Lynch, Phys. Rev. B 54, 6301 (1996).

${ }^{44}$ A. K. Shukla, P. Krüger, R. S. Dhaka, D. I. Sayago, K. Horn, and S. R. Barman, Phys. Rev. B 75, 235419 (2007).

${ }^{45}$ D. C. Langreth, Phys. Rev. B 1, 471 (1970).

${ }^{46}$ B. Unal, C. J. Jenks, and P. A. Thiel, Phys. Rev. B 77, 195419 (2008).

${ }^{47}$ P. Jeglič, M. Heggen, M. Feuerbacher, B. Bauer, P. Gille, and F. Haarmann, J. Alloys Compd. 480, 141 (2009).

${ }^{48}$ M. Mihalkovič and M. Widom, Phys. Rev. B 75, 014207 (2007).

${ }^{49}$ H. R. Sharma, M. Shimoda, V. Fournée, A. R. Ross, T. A. Lograsso, and A. P. Tsai, Phys. Rev. B 71, 224201 (2005).

${ }^{50}$ H. R. Sharma, V. Fournée, M. Shimoda, A. R. Ross, T. A.
Lograsso, A. P. Tsai, and A. Yamamoto, Phys. Rev. Lett. 93, 165502 (2004).

${ }^{51}$ M. Gierer, M. A. Van Hove, A. I. Goldman, Z. Shen, S.-L. Chang, C. J. Jenks, C.-M. Zhang, and P. A. Thiel, Phys. Rev. Lett. 78, 467 (1997).

${ }^{52}$ N. Ferralis, K. Pussi, E. J. Cox, M. Gierer, J. Ledieu, I. R. Fisher, C. J. Jenks, M. Lindroos, R. McGrath, and R. D. Diehl, Phys. Rev. B 69, 153404 (2004)

${ }^{53}$ V. Fournée, E. Belin-Ferré, P. Pêcheur, J. Tobola, Z. Dankhazi, A. Sadoc, and H. Müller, J. Phys.: Condens. Matter 14, 87 (2002).

${ }^{54}$ G. Trambly de Laissardière, D. Nguyen Manh, and D. Mayou, Prog. Mater. Sci. 50, 679 (2005). 